\title{
The validity of a computerized visual searching task as an indicator of brain damage
}

\author{
MICHAEL A. DeMITA \\ Psych Systems, Inc., Baltimore, Maryland 21209 \\ JAMES H. JOHNSON \\ Illinois Institute of Technology, Chicago, Illinois 60616 \\ and \\ KEN E. HANSEN \\ Psych Systems, Inc., Baltimore, Maryland 21209
}

\begin{abstract}
Goldstein, Rennick, Welch, and Shelly (1973) developed a visual searching task (VST) that succeeded in obtaining a hit rate of $94.1 \%$ correct classifications when comparing braindamaged and normal subjects and a $79.4 \%$ hit rate when comparing brain-damaged and psychiatric subjects. Goldstein and Kyc (1978) reported $92.5 \%$ correct classifications for the brain-damaged vs. normal comparisons and $82.5 \%$ correct classifications for brain-damaged vs. schizophrenic comparisons. We computerized the administration of the VST and found $85.7 \%$ correct classifications for the brain-damaged vs. normal groups and $71.4 \%$ correct classifications for the brain-damaged vs. psychiatric group. These results suggest that the computerized VST (CVST) is also a potentially valid indicator of brain damage.
\end{abstract}

Goldstein, Rennick, Welch, and Shelly (1973) reported a visual search task (VST) that succeeded in obtaining a hit rate of $94.1 \%$ correct classifications when comparing brain-damaged and normal subjects and a $79.4 \%$ hit rate when comparing brain-damaged and psychiatric subjects. Goldstein and Kyc (1978) reported $92.5 \%$ correct classifications for the brain-damaged vs. normal comparisons and $82.5 \%$ correct classifications for braindamaged vs. schizophrenic comparisons. We have developed a computerized version of the same task, the CVST. The purpose of this paper is to present a validity study of the CVST to determine whether the computerized version has validity equivalent to that of the VST. The present experiment tests the validity of the CVST as an indicator of brain damage by comparing normal and psychiatric patients with subjects who have confirmed evidence of brain damage. Throughout our study, stringent standards for defining the braindamaged group and control groups were utilized; these were not used in the previous experiments. These standards were partially taken from Malec (1978) and consist of computerized tomographic scans (CATSCAN) for all experimental groups, either confirming or disconfirming brain damage, and MMPIs for the same groups to confirm or disconfirm psychopathology.

The new CVST consists of two sets of checkerboard

Requests for reprints should be sent to Michael A. DeMita, Psych Systems, Inc., Suite 404, 600 Reisterstown Road, Baltimore, Maryland 21209. patterns. The stimuli are presented on the display of a CRT terminal, unlike the projection screens used in the Goldstein (Goldstein \& Kyc, 1978; Goldstein et al., 1973) experiments.

\section{METHOD}

\section{Subjects}

This study used three groups of subjects, consisting of seven brain-damaged patients, seven non-brain-damaged psychiatric patients, and seven nonpatient normal controls. The seven brain-damaged individuals were taken from private practices of clinicians across the United States. All had CATSCAN documentation of brain damage analyzed by qualified psychiatrists or neurologists. All subjects in this group had taken the MMPI and had scores that suggested no psychotic, personality, or emotional disorders. The seven psychiatric subjects consisted of patients drawn from the same varied private practice populations. These subjects all had negative CATSCANs and MMPIs suggesting one of a number of psychotic, neurotic, or personality disorders. The seven normal control subjects were drawn from a random sampling of acquaintances of these same clinicians. CATSCANs were not available for these subjects.

The mean age of the brain-damaged group was 43.29 years $(\mathrm{SD}=12.23)$, the mean age of the psychiatric group was 44.43 years $(\mathrm{SD}=12.97)$, and the mean age of normal group was 43.43 years $(S D=12.38)$. The mean number of years of education for the brain-damaged group was 13.71 ( $\mathrm{SD}=3.09$ ), 11.71 $(\mathrm{SD}=2.43)$ for the psychiatric group, and $12.43(\mathrm{SD}=2.23)$ for the normal group. The results of an analysis of variance indicated no significant differences in age $[\mathrm{F}(2,18)=.017$, $p>.05]$ or in education $[F(2,18)=1.06, p>.05]$ for the groups. In order not to bias the results, brain-damaged subjects with vision problems, lesions in the visual cortex, severely deteriorated advanced neuropathological impairment, or reading impairments were excluded. Table 1 shows the various diagnoses of the brain-damaged group. 
Table 1 Diagnoses of Brain-Damaged Group

1. Closed head injury, left frontal lobe

2. Closed head injury, right frontal lobe

3. Alcoholic organic brain syndrome, diffuse

4. Organic brain syndrome, right occipital, head trauma

5. Cerebrovascular accident, right parietal

6. Organic brain syndrome, right parietal, secondary to CVA

7. Cerebrovascular accident, right hemisphere

\section{Procedure}

Two practice trials were administered to familiarize the subjects with procedure, stimuli, and key pad. The first of these consisted of two square grids surrounding a target in linear fashion. One trial was administered for the first practice set. The second practice set consisted of three trials.

The test apparatus consisted of a Digital Equipment Corporation LSI-11/03 computer with floppy-disk drives and a VT-100 CRT display terminal. Presentation of the stimulus took place on the display terminal. Two stimulus arrays were used. Both arrays consisted of 28 white squares and 28 black squares arranged in checkerboard fashion (see Figure 1). The white squares were divided into a 5 by 5 array of 25 sections. Two sections were missing from each white square at a variety of locations, but never at the same location within the same stimulus pattern. Of the 28 white squares, the center square always represented the target grid and was marked by an arrow ( $\leftrightarrow)$. The other 27 squares were numbered 1-27 with small numbers placed to the center right of the grid. The position of the two holes in the target grid corresponded to the positions of two holes on one of the other numbered grids. Targets were selected from each of the four quadrants of the stimulus pattern to balance the location of the correct matching grid.

The subject was seated with his/her head at a comfortable distance, approximately $40 \mathrm{~cm}$ from the terminal screen. The stimulus image on the display screen was $18 \mathrm{~cm}$ wide $\times 12 \mathrm{~cm}$ high. The subject viewed the stimulus pattern from this position and responded on a small numeric key pad.

During the two practice trials, the subjects were instructed both by a supervisor verbally and by instructions written on the CRT. Subjects were told to look at the target grid and then to look at the rest of the stimulus. Instructions pointed out that only one of the surrounding patterns matched the target grid. A response was recorded by the subjects pressing the number of the pattern that matched the target pattern and then pressing the ENTER key. If the answer was incorrect, a bell sounded and

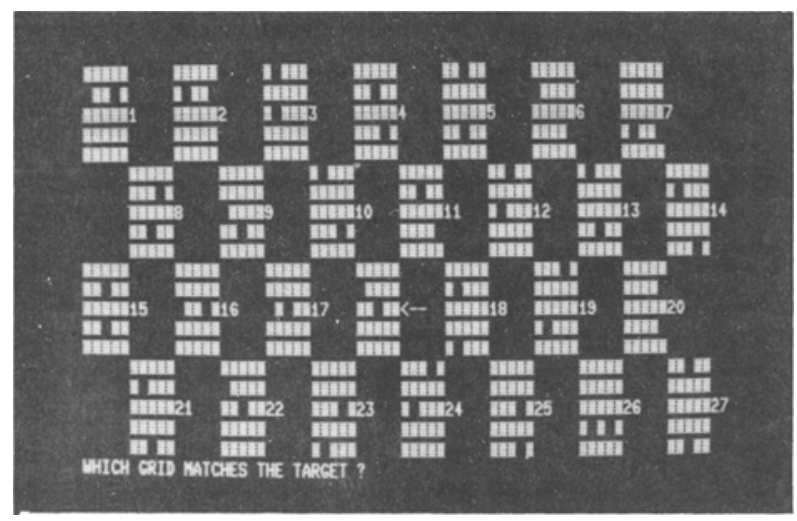

Figure 1. Stimulus display with 28 squares with missing sections. The center square represents the target grid (always marked by an arrow). Other squares are numbered 1-27. the words "INCORREGT-TRY AGAIN" appeared on the screen. If the answer was correct, a new target was placed in the position of the old automatically. Ten targets were presented for each of the two stimulus patterns.

Dependent measures were time and errors for each trial and total performance time (TPT) and total errors for all 20 trials.

\section{RESULTS}

Significant differences were found among groups using TPT as a dependent measure $[F(2,18)=5.37$, $\mathrm{p}<.05]$. The mean for the brain-damaged group was $843.57 \mathrm{sec}(\mathrm{SD}=423.722)$, the psychiatric group mean was $518.00 \mathrm{sec}(\mathrm{SD}=195.878)$, and the normal control group mean was $360.29 \mathrm{sec}(\mathrm{SD}=140.283)$. Goldstein et al. (1973) found that the brain-damaged group obtained a mean time of $641.29 \mathrm{sec}(\mathrm{SD}=250.88)$, the non-brain-damaged psychiatric group obtained a mean time of $398.59 \mathrm{sec}(\mathrm{SD}=163.37)$, and the normal group obtained a mean time of $287.29(\mathrm{SD}=61.79)$. Goldstein and Kyc (1978) found that the brain-damaged group's mean time was $770.6 \mathrm{sec}(\mathrm{SD}=254.04)$, the schizophrenic group's mean time was $529.85 \mathrm{sec}(\mathrm{SD}=$ 203.24), and the normal group's mean time was $315.9 \mathrm{sec}$ $(\mathrm{SD}=73.28)$. Differences in apparatus and stimuli undoubtedly account for mean times' being different for the CVST and the VST.

The scores were ranked, and an optimal cutoff point was found at $375 \mathrm{sec}$. When this cutoff was used to discriminate between brain-damaged and psychiatric subjects, four misclassifications were made, which were all false-positive. Using the same cutoff to discriminate brain-damaged from normal subjects, only two misclassifications were made, both false-positive. Percentages of correct classifications were $85.7 \%$ for the braindamaged vs. normal groups and $71.4 \%$ for the braindamaged vs. psychiatric groups. Goldstein et al. (1973) found their cutoff made two false-negative misclassifications for the normal vs. brain-damaged comparison. Five false-positive misclassifications and two falsenegative misclassifications were made for the braindamaged vs. psychiatric comparison. The hit rates were $94.1 \%$ and $79.4 \%$ for the two comparisons, respectively. Goldstein and Kyc (1978) found that the new cutoff obtained in this experiment produced three false. negative misclassifications for the brain-damaged vs. normal comparison. Three false-negative and four false-positive misclassifications were made for the brain-damaged vs. schizophrenic comparison. The hit rates in this second experiment were $92.5 \%$ and $82.5 \%$ for the two comparisons, respectively.

To determine if the cumulative effect of age or education influenced the TPT. Pearson's product-moment correlations were calculated between age and TPT and between education level and TPT. For age, the correlation coefficient was .04 for all subjects. For education. the coefficient was -.12 . These scores are not signifi- 
cantly different from zero ( $p>.10$ in all cases). These statistics show that it is unlikely that intergroup differences in TPT can be attributed to any cumulative effect age and education may have produced.

\section{DISCUSSION}

The results of the study show that the CVST, like the VST, is a good discriminator of brain-damaged and non-brain-damaged individuals. Differences in the total performance time of psychiatric and normal groups may be due to brain dysfunction undiagnosable by present methods, or, as Goldstein et al. (1973) have pointed out, these differences may be attributable to the effects of medication, which were not controlled for.

The purpose of this study was to determine if the CVST is as good an indicator of brain damage as the VST is. Although the sample size used in this experiment was not as large as the size used in the Goldstein and Kyc (1978) and Goldstein et al. (1973) studies, good results were obtained for this anchor study. This experiment made use of stringent procedures in the definition of brain-damaged subjects (i.e., CATSCANs) and psychiatric subjects (i.e., MMPIs), while still supporting the validity of the VST as an indicator of brain damage.

\section{REFERENCES}

Goldstein, G., \& Kyc, F. Performance of brain-damaged, schizophrenic and normal subjects on a visual searching task. Perceptual and Motor Skills, 1978, 46, 731-734.

Goldstein, G., Rennick, P. M., Welch, R. B., \& Shelly, C. H. The validity of a visual searching task as an indicator of braindamage. Journal of Consulting and Clinical Psychology, 1973, 41, 434-437.

MALEC, J. Neuropsychological assessment of schizophrenia versus brain damage: A review. Journal of Nervous and Mental Disease, 1978, 166, 507-516. 